\title{
SINTESE DA FASE SODALITA COM O USO DO CAULIM DA REGIÃO BORBOREMA-SERIDÓ (RN/PB) ATIVADO TERMICAMENTE
}

C. G. M Santos ${ }^{2,3}$; P. M. P. B. Lana ${ }^{2,3}$, A. A. S. Silva ${ }^{1,3}$; C. N Barbato ${ }^{2}$; J. A. Sampaio ${ }^{3}$; F. M. S Garrido ${ }^{1}$; F. A. N. G. Silva ${ }^{1}$ ${ }^{1}$ Instituto de Química - Universidade Federal do Rio de Janeiro

${ }^{2}$ Escola de Química - Universidade Federal do Rio de Janeiro

${ }^{3}$ Centro de Tecnologia Mineral - MCTI cgsantos@cetem.gov.br; fnogueira@iq.ufrj.br

Artigo submetido em Setenbro/2012 e aceito em Novembro/2012

\section{RESUMO}

A fase mineral sodalita foi obtida com sucesso a partir de reações hidrotérmicas com o uso de caulim proveniente da região Borborema-Seridó (RN/PB), hidróxido e carbonato de sódio. Desse modo, o caulim, com granulometria menor que $37 \mu \mathrm{m}$, foi tratado inicialmente em diferentes temperaturas. A calcinação foi realizada nas temperaturas de 500,700 e $900^{\circ} \mathrm{C} \mathrm{com}$ o objetivo de formar a metacaulinita. Os produtos calcinados foram submetidos à síntese hidrotermal por 1 e 4h, no qual foram utilizados $\mathrm{Na}_{2} \mathrm{CO}_{3}$ e $\mathrm{NaOH}$. De acordo com os resultados, nos caulins calcinados a $700 \mathrm{e}$ $900^{\circ} \mathrm{C}$ ocorreu a completa transição de fase da caulinita para a metacaulinita e, para a síntese no qual utilizou o caulim calcinado a $700^{\circ} \mathrm{C}$ ocorreu a formação da fase zeolítica sodalita básica caracterizada por difração de raios $X$ e espectroscopia vibracional no infravermelho que mostrara picos e bandas intensas e definidas indicando maior cristalinidade do material.

PALAVRAS-CHAVE: Caulim; Síntese Hidrotermal; Sodalita

\section{SINTHESIS OF SODALITE'S PHASE WITH THE USE OF KAOLIN FROM BORBOREMA-SERIDÓ REGION (RN/PB) THERMALLY ACTIVATED}

\begin{abstract}
Sodalite's mineral phase was successfully obtained by hydrothermal reactions with the use of kaolin from the Borborema-Seridó (RN/PB) region, $\mathrm{NaOH}$ and $\mathrm{Na}_{2} \mathrm{CO}_{3}$. Therefore, Kaolin lower than $37 \mu \mathrm{m}$ was used with previous thermal treatment. Calcination was carried out in the temperatures of 500,700 and $900^{\circ} \mathrm{C}$ in order to form metakaolinite. Hydrothermal synthesis by 1 and 4 hours was carried out with the calcined product.
\end{abstract}

According to results, on calcinated kaolins at 700 and $900^{\circ} \mathrm{C}$ occurred the complete transition of kaolinite phase to metakaolinite and, in the synthesis with the use of kaolin calcined at $700^{\circ} \mathrm{C}$, the sodalite basic phase was formed and characterized by $X$-ray diffraction and infrared vibrational spectroscopy which showed peaks and bands more intense and definite indicating the high crystallinity of this material.

KEY-WORDS: Kaolinite, Hydrotermal Synthesis, Sodalite. 


\section{SINTESE DA FASE SODALITA COM O USO DO CAULIM DA REGIÃO BORBOREMA-SERIDÓ (RN/PB) ATIVADO TERMICAMENTE}

\section{INTRODUÇÃO}

As zeólitas podem ser definidas como aluminossilicatos cristalinos com uma estrutura tridimensional composta por um conjunto de cavidades ocupadas por íons e moléculas de água, ambos com considerável liberdade de movimento, permitindo a troca iônica e uma hidratação reversível (SHINZATO, 2007). A troca iônica e a inclusão de moléculas, nas cavidades, só são possíveis para aquelas moléculas cujas dimensões são inferiores a um valor crítico, que varia de uma zeólita para outra (RIGO E PERGHER, 2009).

As potenciais aplicações das zeólitas, no abrandamento de água dura, tratamento de esgotos, melhoramento de solos e adsorção de metais pesados e de óleos, têm sido bastante estudadas, devido ao baixo custo, a fácil obtenção e a possibilidade de reutilização destes materiais (HELLER-KALLAI E LAPIDES, 2007). No entanto, como as zeólitas naturais não conseguem suprir a grande demanda industrial, se tornou uma necessidade a síntese das mesmas para viabilizar seu uso em grande escala na indústria (RESENDE et al., 2008)

A sodalita básica, $\mathrm{Na}_{8}\left[\mathrm{SiAlO}_{4}\right]_{6}(\mathrm{X})_{2} \cdot 2 \mathrm{H}_{2} \mathrm{O}$ sendo $\mathrm{X}=\mathrm{OH}^{-}, \mathrm{CO}_{3}{ }^{2-}, \mathrm{Cl}^{-}, \mathrm{SO}_{4}{ }^{2-}$, é considerada um material zeolítico devido a sua estrutura porosa e as moléculas de água nas cavidades, apresentando, desta forma alta capacidade de adsorção e de troca catiônica (PAZ et al., 2010).

Neste contexto, o objetivo deste trabalho foi investigar um procedimento de síntese que resulte na obtenção da zeólita sintética sodalita básica a partir do caulim da região Borborema-Seridó a fim de compreender, em trabalhos futuros, a influência dessa fase no processo de adsorção de metais pesados.

\section{MATERIAIS E MÉTODOS} Síntese

Para síntese hidrotermal da sodalita básica foi utilizado como fonte de alumínio e de silício o caulim da região Borborema-Seridó (RN/PB). Desse modo uma alíquota homogênea de caulim foi peneirada a úmido em um peneirador vibratório (684,5 rpm) equipado com peneiras de abertura de 74 e $37 \mu \mathrm{m}$. A fração menor que $37 \mu \mathrm{m}$ foi filtrada, seca em estufa, quarteada e encaminhada a caracterização por absorção atômica e ao tratamento térmico a 500,700 e $900^{\circ} \mathrm{C}$ por $2 \mathrm{~h}$. Como fontes de $\mathrm{Na}$ foram utilizados $\mathrm{Na}_{2} \mathrm{CO}_{3}$ e $\mathrm{NaOH}$. As condições estequiométricas dos reagentes utilizados neste estudo encontram-se detalhadas na Tabela 1.

Tabela 1. Condições estequiométricas utilizadas na síntese da sodalita.

\begin{tabular}{|c|c|c|c|c|}
\hline Reagentes & Caulim: $\mathrm{Al}_{2} \mathrm{Si}_{2} \mathrm{O}_{5}(\mathrm{OH})_{4}$ & $\mathrm{Na}_{2} \mathrm{CO}_{3}$ & $\mathrm{NaOH}$ & $\mathrm{H}_{2} \mathrm{O}$ \\
\hline Número de mols & 1,0 & 8,0 & 1,9 & 143,3 \\
\hline
\end{tabular}


A digestão foi realizada em vasos de pressão de aço inox que foram colocados em forno com rampa de aquecimento de $10^{\circ} \mathrm{C}$ por minuto até atingir a temperatura de $170^{\circ} \mathrm{C}$. Ao atingir esta temperatura os vasos foram mantidos sob aquecimento por 1 e $4 \mathrm{~h}$. Decorrido o tempo de síntese, os vasos foram resfriados com água corrente e o produto formado foi seco em estufa, em temperaturas próximas a $80^{\circ} \mathrm{C}$ e encaminhados à difração de raios $\mathrm{X}$ e a espectroscopia vibracional no infravermelho.

\section{Caracterização}

A análise química do caulim com granulometria inferior a $37 \mu \mathrm{m}$ foi feita por absorção atômica no equipamento AA6 Varian, com comprimento de onda de $248,3 \mathrm{~nm}$, fenda de 0,5 $\mathrm{nm}$ e com ar/acetileno.

Os difratogramas de raios $X$ foram obtidos pelo método do pó. As análises foram realizadas no equipamento Bruker-AXS D5005, radiação $\mathrm{Cu} K \alpha(40 \mathrm{kV} / 20 \mathrm{~mA})$ para os caulins bruto e calcinados e Co $\mathrm{K} \alpha(35 \mathrm{kV} / 40 \mathrm{~mA})$, velocidade do goniômetro de $0,02^{\circ}(2 \theta)$ por passo com tempo de contagem de $1 \mathrm{~s}$ por passo e coletados de 5 a $80(2 \theta)$.

A análise por espectroscopia vibracional no infravermelho foi realizada no espectrofotômetro com transformada de Fourier, marca Nicolet Magna, com registros de 4000 a $400 \mathrm{~cm}^{-1}$, resolução de $4 \mathrm{~cm}^{-1}$, em pastilhas de $\mathrm{KBr}$.

\section{RESULTADOS E DISCUSSÃO}

\section{Caracterização do Caulim}

O caulim, com granulometria inferior a $37 \mu \mathrm{m}$, foi submetido à análise química por absorção atômica. A avaliação dos resultados, ilustrados na Tabela 2, indica que o caulim da região Borborema-Seridó é essencialmente caulinítico, já que a composição química encontrada para $\mathrm{SiO}_{2}$ e $\mathrm{Al}_{2} \mathrm{O}_{3}, 45,9$ e 39,2\% respectivamente, se aproxima da composição química teórica da caulinita $(46,54$ e $39,50 \%)$.

Desse modo, o caulim foi submetido à calcinação em diferentes temperaturas com o objetivo de transformá-lo em metacaulinita e, assim, tornar os átomos de silício e de alumínio mais disponíveis para síntese da sodalita (PAZ et al., 2010). No entanto, é importante ressaltar, que a reatividade da metacaulinita formada varia de acordo com a temperatura de calcinação e do tipo de caulim utilizado, sendo que, de acordo com as impurezas que o material possa conter, este tratamento térmico poderá contribuir diferentemente na formação de zeólitas (HELLER-KALLAI E LAPIDES, 2007).

Com o objetivo de avaliar o resultado do tratamento térmico submetido às amostras de caulim, a difratometria de raios $X$ (DRX) foi utilizada por ser a principal técnica na identificação mineralógica de amostras de caulim. A análise dos resultados, Figura 1, indica que este caulim é caulinítico, uma vez que apresentam picos referentes ao mineral caulinita, isto é, 14,33 e 28,94 (20). Esse resultado corrobora com os obtidos por meio da análise química por absorção atômica. No entanto, no difratograma, também são observados os picos

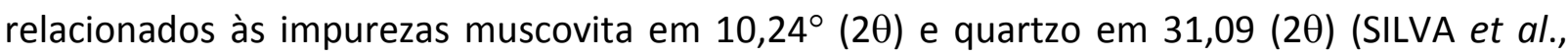
2011). 
Tabela 2 - Resultados da análise química por absorção atômica para o caulim com granulometria inferior a $-37 \mu \mathrm{m}$.

\begin{tabular}{|c|c|c|c|}
\hline Óxidos & $-37 \mu \mathrm{m}(\%)$ & Óxidos & $-37 \mu \mathrm{m}(\%)$ \\
\hline $\mathrm{SiO}_{2}$ & 51,60 & $\mathrm{MgO}$ & 0,05 \\
\hline $\mathrm{Al}_{2} \mathrm{O}_{3}$ & 36,32 & $\mathrm{~K}_{2} \mathrm{O}$ & 0,71 \\
\hline $\mathrm{Fe}_{2} \mathrm{O}_{3}{ }^{*}$ & 0,26 & $\mathrm{Na}_{2} \mathrm{O}$ & 0,73 \\
\hline $\mathrm{TiO}_{2}$ & $<0,01$ & $\mathrm{P}_{2} \mathrm{O}_{5}$ & 0,02 \\
\hline $\mathrm{Cr}_{2} \mathrm{O}_{3}$ & $<0,01$ & $\mathrm{Zr}_{2} \mathrm{O}$ & $<0,3$ \\
\hline $\mathrm{MnO}$ & $<0,01$ & $\mathrm{PF}$ & 13,75 \\
\hline $\mathrm{CaO}$ & 0,07 & $\begin{array}{l}\text { PF }=\text { Perda ao Fogo } \\
\text { *Teor de Fe convertido a } \mathrm{Fe}_{2} \mathrm{O}_{3}\end{array}$ \\
\hline
\end{tabular}
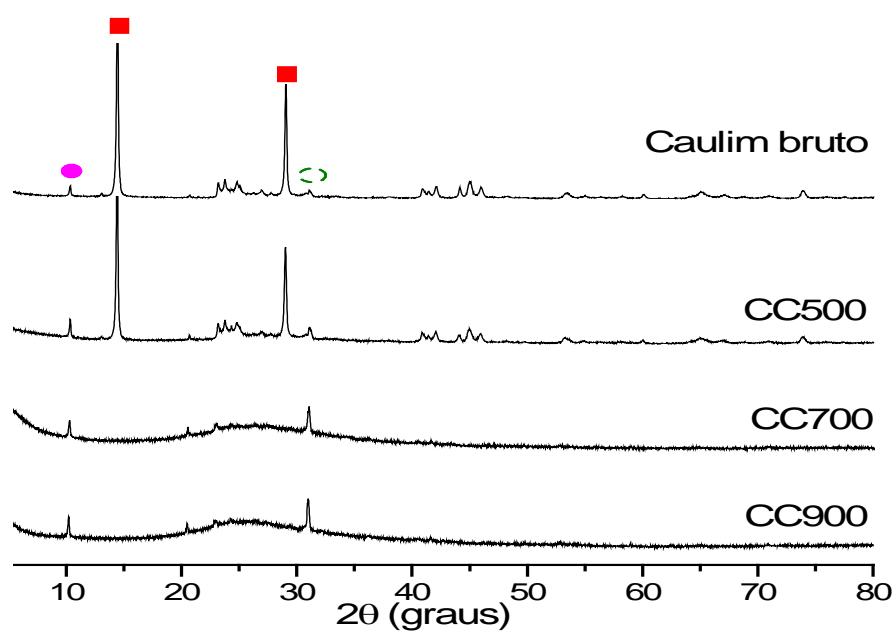

Caulinita Muscovita $\Leftrightarrow$ Quartzo

Figura 1. Difratogramas de raios $X, C o K \alpha$, das amostras de caulim bruto e calcinado a 500, 700 e $900^{\circ} \mathrm{C}$.

Conforme observado, Figura 1 , no caulim calcinado a $500^{\circ} \mathrm{C}$ a estrutura cristalina da caulinita não foi totalmente rompida pela desidroxilação, pois, ainda são observados os picos referentes a este mineral. Já os caulins calcinados a 700 e $900^{\circ} \mathrm{C}$ houve o total rompimento da estrutura cristalina da caulinita formando-se um material amorfo, ou seja, a metacaulinita.

\section{Caracterização dos produtos da síntese}


A síntese de zeólitas bem como a sua cristalização, também é dependente do $\mathrm{pH}$ do meio reacional, que deve variar entre 8 e 13 e por este motivo são utilizados reagentes alcalinos, uma vez que a alcalinidade favorece a solubilização dos aluminosilicatos amorfos tornando-os, desse modo, mais reativos (MIGNONI, 2012). Neste contexto, buscou-se uma relação entre $\mathrm{NaOH}$, mais usado, e $\mathrm{Na}_{2} \mathrm{CO}_{3}$, mais viável economicamente, de modo que tornase a síntese mais acessível.

Os tempos de calcinação do caulim e o de síntese foram os parâmetros avaliados na formação da zeólita sodalita básica. Assim, por meio dos difratogramas de raios X, Figura 2, observa-se que o caulim calcinado a $500^{\circ} \mathrm{C}$ utilizado na síntese de $1 \mathrm{~h}$ contêm picos referentes a sodalita básica, 14,05, 24,50 e 43,04 (20) (IZA ON LINE, MINERALOGY DATABASE, CRYSTMET). No entanto, os picos relacionados ao mineral caulinita encontram-se intensos e definidos, além de se observar o pico relacionado à muscovita, o que indica que o caulim e o tempo de síntese não são indicados a obtenção da fase zeolítica pura. Já o difratograma do caulim calcinado a $700^{\circ} \mathrm{C}$ no mesmo tempo de síntese, não são observados os picos relacionados à caulinita e a muscovita, o que indica a total conversão das fases à formação da sodalita. Na síntese em que fez-se o uso do caulim calcinado a $900^{\circ} \mathrm{C}$, além da fase sodalita, também, é observado uma fase intermediária em 15,55 (20).

Ao compararmos os DRX das sínteses realizadas em 1 e em $4 \mathrm{~h}$ para os caulins calcinados a 500,700 e $900^{\circ} \mathrm{C}$, observa-se que nas sínteses no qual o tempo foi maior, os picos referentes a sodalita básica são mais definidos e finos indicando que $4 \mathrm{~h}$ de síntese é mais favorável a formação da fase com um bom grau de cristalinidade. Estes resultados estão de acordo com a literatura na qual descreve que a zeólita sodalita básica é a fase mais estável e compacta dentre as estruturas zeolíticas em comum, tais como, a zeólita (A), a zeólita $X / Y$ (FAU) e a EMT além, da hidrossodalita. Cabe ressaltar, que os tempos de reação de nosso trabalho são menores do que os descritos na maioria dos trabalhos que realizaram a síntese da sodalita a partir de caulim (HERMELER et al., 1991, BUHL et al., 1997, PAZ et al., 2010, FREITAS, 2011). No entanto, ao comparar os difratogramas de 500,700 e $900^{\circ} \mathrm{C}$, observa-se que a muscovita, ainda, é encontrada na síntese com o uso do caulim calcinado a $500^{\circ} \mathrm{C}$. 


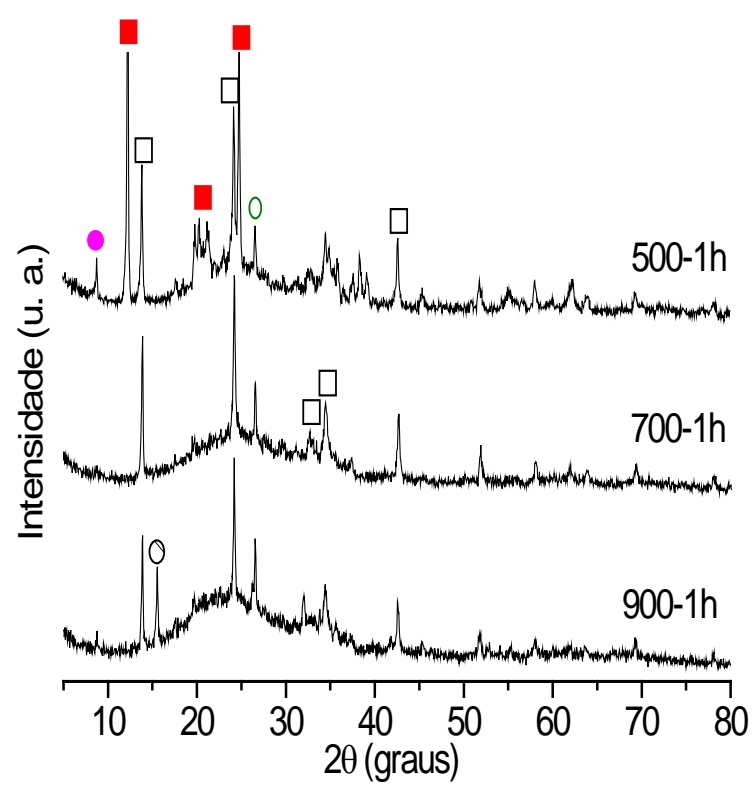

$\square$ Sodalita Básica・ Muscovita OFase Intermediária

Caulinita $\quad 0$ Quartzo

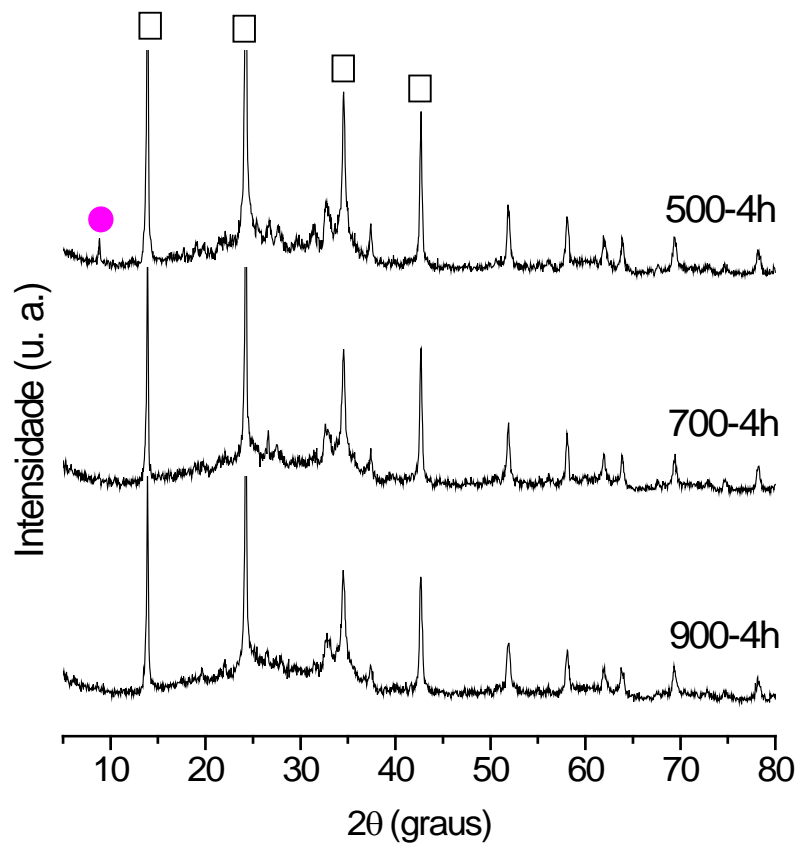

Sodalita Básica Muscovita

Figura 2. Difratogramas de raios $\mathrm{X}, \mathrm{Cu} K \alpha$, das fases sintetizadas com o uso de caulins calcinados a 500, 700 e $900^{\circ} \mathrm{C}$. Em (A) $1 \mathrm{~h}$ e em (B) $4 \mathrm{~h}$.

A espectroscopia vibracional no infravermelho foi utilizada como técnica complementar à DRX. As análises por IV das amostras sintetizadas por $4 \mathrm{~h}$, Figura 3 , indicam que somente na síntese na qual foi utilizado o caulim calcinado a $700^{\circ} \mathrm{C}$ é possível formar a sodalita básica uma vez que as bandas relacionadas a esta fase, 990, 690, 660, 560, $430 \mathrm{~cm}^{-1}$ (HERMELER et al., 1991, HELLER-KALLAI E LAPIDES, 2007, PAZ et al., 2010) encontram-se bem definidas. Estes resultados estão de acordo com os obtidos por meio da DRX indicando que esta zeólita encontra-se cristalizada e não há a existência de uma fase intermediária. Dentre as espécies contidas dentro das cavidades destacam-se a $\mathrm{OH}^{-}$com bandas entre 3.400 e 3.600 $\mathrm{cm}^{-1}$, a $\mathrm{H}_{2} \mathrm{O}$ observada próxima a $1.650 \mathrm{~cm}^{-1}$ e o $\mathrm{CO}_{3}{ }^{2-}$ em 1.450 e $1.410 \mathrm{~cm}^{-1}$. 


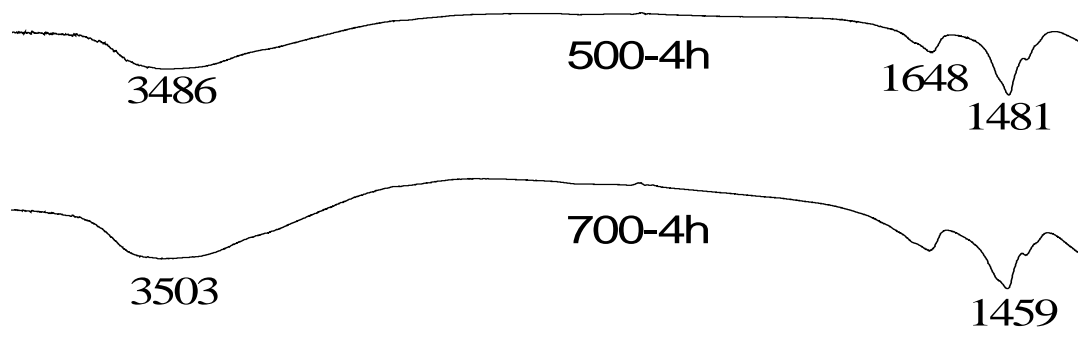

3486

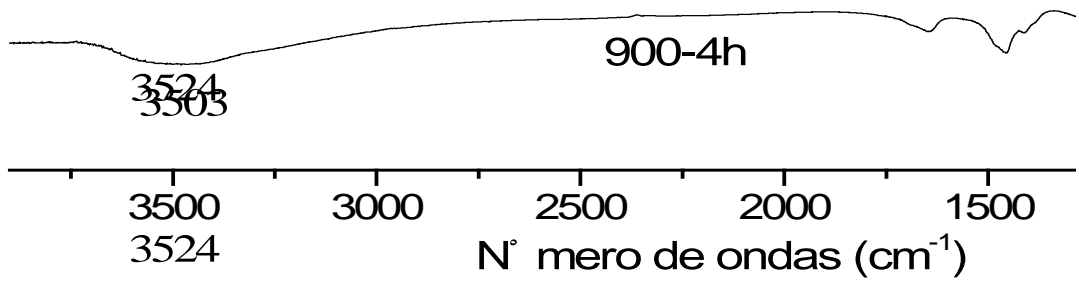

Figura 3. Espectros no infravermelho das sínteses por $4 \mathrm{~h}$ com o uso de caulins calcinados a 500,700 e $900^{\circ} \mathrm{C}$.

\section{CONCLUSÕES}

De acordo com os resultados apresentados, pode-se concluir que é possível sintetizar a sodalita básica a partir do caulim bruto, da região Borborema-Seridó, calcinado a $700^{\circ} \mathrm{C}$, com tempo de síntese de $4 \mathrm{~h}$ a $170^{\circ} \mathrm{C}$ com o uso de $\mathrm{NaOH}$ e $\mathrm{Na}_{2} \mathrm{CO}_{3}$ na razão 1:4,25.

\section{AGRADECIMENTOS}

Os autores agradecem a FAPERJ pelo auxílio financeiro (Processo: INST - 110.794/2012) e ao CNPq pela bolsa de Iniciação Científica.

\section{REFERÊNCIAS BIBLIOGRÁFICAS}

1. SHINZATO, M. C., Remoção de Metais Pesados em Solução por Zeólitas Naturais: Revisão Crítica, Revista do Instituto Geológico 27-28, P. 65-78, 2007.

2. RIGO, R. T., PERGHeR, S. B. C. Um Novo Procedimento de Síntese da Zeólita a Empregando Argilas Naturais, Química Nova, vol. 21, n 1, pag. 21-25, 2009.

3. HELLER-KALLAI, L.; LAPIDES, I. Reactions of Kaolinites and metakaolinites with $\mathrm{NaOH}$ comparison of different samples (Part 1). Applied Clay Science, v. 35, p. 99-107, 2007.

4. RESENDE, N. G. A. M, MONTE, M. B. M., PAIVA, P. R. P. Bauxita. In: Luz. A. B., Lins, F. A. F. Rochas e minerais industriais, 2a Ed., Cap. 39, 889-916, 990p. CETEM/MCT, Rio de Janeiro, 2008. 
5. PAZ, S. P. A., ANGÉLICA, R. S., NEVES, R. F. Síntese Hidrotermal de Sodalita a partir de um Rejeito de Caulim Termicamente Ativado. Química Nova, v. 33, n. 3, p. 579-583, 2010.

6. SILVA, F. A N. G, Garrido, F. M. S., MEDEIROS, M. E., SAMPAIO, J. A., LUZ, A. B., MELLO, L. S., SILVA, F. T. Alvejamento químico de caulins Brasileiros: efeito do potencial eletroquímico da polpa e do ajuste do pH. Química Nova, v. 34, n. 2, p. 262-267, 2011.

7. MIGNONI, M. L., Zeólitas obtidas com líquidos iônicos como direcionadores de estrutura: síntese e reatividade. Março 2012. 168 f. Tese (Doutorado em Química) - Instituto de Química, Universidade Federal do Rio Grande do Sul, 2011.

8. www.iza-online.org, consultado em setembro de 2012.

9. webmineral.com, consultado em setembro de 2012.

10. Crystmet em www.periodicocapes.com.br, consultado em setembro de 2012.

11. HERMELER, G.; BUHL, J. CH., HOFFMANN, W. The Influence of Carbonate on the Synthesis of an ntermediate Phase Between sodalite and Cancrinite. Catalysis Today, p. 415426, 1991.

12. BUHL, J. $\mathrm{CH}$, hOFFMANN, W., BUCKERMANN, W. A., MIILleR-WARMUTH, W. The crystallization kinetics of sodalites grown by the hydrothermal transformation of kaolinite studied by ${ }^{29}$ Si MAS NMR. Solid State Nuclear Magnetic Resonance, 9, pag. 121-128, 1997.

13. FREITAS, V. A. A.; LIMA, J. S. V.; COUCEIRO, P. R. C. Caracterização e análise estrutural da hidroxisodalita sintetizada a partir de amostras de solo amazônico. Cerâmica, v. 57, n. 343, p. 281- 287, 2011. 